\title{
Hermitian Toeplitz determinants of the second and third-order for classes of close-to-star functions
}

\section{Piotr Jastrzębski ${ }^{1}$. Bogumiła Kowalczyk ${ }^{1}$ - Oh Sang Kwon ${ }^{2}$ - Adam Lecko ${ }^{1}$ (D) Young Jae $\mathrm{Sim}^{2}$}

Received: 22 October 2019 / Accepted: 30 June 2020 / Published online: 10 July 2020

(c) The Author(s) 2020

\section{Abstract}

For some subclasses of close-to-star functions the sharp upper and lower bounds of the second and third-order Hermitian Toeplitz determinants are computed.

Keywords Hermitian Toeplitz determinant - Univalent functions · Close-to-star functions · Carathéodory class

Mathematics Subject Classification 30C45 · 30C50

\section{Introduction}

Given $r>0$, let $\mathbb{D}_{r}:=\{z \in \mathbb{C}:|z|<r\}, \mathbb{T}_{r}:=\{z \in \mathbb{C}:|z|=r\}, \mathbb{D}:=\mathbb{D}_{1}, \mathbb{T}:=\mathbb{T}_{1}$ and $\overline{\mathbb{D}}:=\{z \in \mathbb{C}:|z| \leq 1\}$. Let $\mathcal{H}$ be the class of all analytic functions in $\mathbb{D}$ and $\mathcal{A}$ be its subclass of functions $f$ normalized by $f(0)=0$ and $f^{\prime}(0)=1$, i.e., of the form

$$
f(z)=\sum_{n=1}^{\infty} a_{n} z^{n}, \quad a_{1}:=1, \quad z \in \mathbb{D} .
$$

Let $\mathcal{S}$ be the subclass of $\mathcal{A}$ of all univalent functions.

\footnotetext{
Adam Lecko

alecko@matman.uwm.edu.pl

Piotr Jastrzębski

piojas@matman.uwm.edu.pl

Bogumiła Kowalczyk

b.kowalczyk@matman.uwm.edu.pl

Oh Sang Kwon

oskwon@ks.ac.kr

Young Jae Sim

yjsim@ks.ac.kr

1 Department of Complex Analysis, Faculty of Mathematics and Computer Science, University of Warmia and Mazury in Olsztyn, ul. Słoneczna 54, 10-710 Olsztyn, Poland

2 Department of Mathematics, Kyungsung University, Busan 48434, Korea
} 
Given $q, n \in \mathbb{N}$, the Hermitian Toeplitz determinant $T_{q, n}(f)$ of $f \in \mathcal{A}$ of the form (1) is defined by

$$
T_{q, n}(f):=\left|\begin{array}{cccc}
a_{n} & a_{n+1} & \ldots & a_{n+q-1} \\
\bar{a}_{n+1} & a_{n} & \ldots & a_{n+q-2} \\
\vdots & \vdots & \vdots & \vdots \\
\bar{a}_{n+q-1} & \bar{a}_{n+q-2} & \ldots & a_{n}
\end{array}\right|,
$$

where $\bar{a}_{k}:=\overline{a_{k}}$.

Recently, Ali, Thomas and Vasudevarao [2] introduced the concept of the symmetric Toeplitz determinant $T_{q}(n)$ for $f \in \mathcal{A}$ in the following way:

$$
T_{q}(n)[f]:=\left|\begin{array}{cccc}
a_{n} & a_{n+1} & \ldots & a_{n+q-1} \\
a_{n+1} & a_{n} & \ldots & a_{n+q-2} \\
\vdots & \vdots & \vdots & \vdots \\
a_{n+q-1} & a_{n+q-2} & \ldots & a_{n}
\end{array}\right| .
$$

They found estimates for $T_{2}(n), T_{3}(1), T_{3}(2)$, and $T_{2}(3)$ over selected subclasses of $\mathcal{A}$.

In recent years many papers have been devoted to the estimation of determinants whose entries are coefficients of functions in the class $\mathcal{A}$ or its subclasses. Hankel matrices i.e., square matrices which have constant entries along the reverse diagonal and the generalized Zalcman functional $J_{m, n}(f):=a_{m+n-1}-a_{m} a_{n}, m, n \in \mathbb{N}$, are of particular interest (see e.g., $[3,4,9-11,18,20,22-25,28,32,34,35,39])$.

In [14,21], research was instigated into the study of Hermitian Toeplitz determinants which elements are coefficients of functions in subclasses of $\mathcal{A}$, observing that Hermitian Toeplitz matrices play an important role in functional analysis, applied mathematics as well as in technical sciences. In this paper we continue this research by finding the sharp upper and lower bounds of the second and third-order Toeplitz determinants over subclasses of close-to-star functions.

Let $\mathcal{F}$ be a subclass of $\mathcal{A}$ such that $\mathcal{F}(2):=\left\{f \in \mathcal{F}: a_{2}=0\right\}$ is a nonempty subfamily and $A_{2}(\mathcal{F}):=\max \left\{\left|a_{2}\right|: f \in \mathcal{F}\right\}$ exists. Since for $f \in \mathcal{A}$,

$$
T_{2,1}(f)=1-\left|a_{2}\right|^{2},
$$

the result below is clear. Equality for the lower bound is attained by a function in $\mathcal{F}$ which is extremal for $A_{2}(\mathcal{F})$. Each function from $\mathcal{F}(2)$ makes equality for the upper bound.

Theorem 1 Let $\mathcal{F}$ be a subclass of $\mathcal{A}$ such that $\mathcal{F}(2) \neq \emptyset$ and $A_{2}(\mathcal{F})$ exists. Then

$$
1-A_{2}^{2}(\mathcal{F}) \leq T_{2,1}(f) \leq 1 \text {. }
$$

Both inequalities are sharp.

Let $\mathcal{S}^{*}$ be the subclass of $\mathcal{S}$ of starlike functions ([1]), i.e., $f \in \mathcal{S}^{*}$ if $f \in \mathcal{A}$ and

$$
\operatorname{Re} \frac{z f^{\prime}(z)}{f(z)}>0, \quad z \in \mathbb{D} \text {. }
$$

A function $f \in \mathcal{A}$ is called close-to-star if there exist $g \in \mathcal{S}^{*}$ and $\beta \in \mathbb{R}$ such that

$$
\operatorname{Re} \frac{\mathrm{e}^{\mathrm{i} \beta} f(z)}{g(z)}>0, \quad z \in \mathbb{D} .
$$

The class $\mathcal{C S} \mathcal{T}$ of all close-to-star functions which was introduced by Reade [36] bear the same relation to the class of close-to-convex functions as the class of starlike functions bear to 
the class of convex functions ([36, p. 61]). This relationship is called Alexander type relation ([1]). Namely, $f \in \mathcal{C S} \mathcal{T}$ if, and only if, a function

$$
F(z):=\int_{0}^{z} \frac{f(t)}{t} d t, \quad z \in \mathbb{D},
$$

is close-to-convex ([19], [16, Vol. II, p. 3]). The class of close-to-convex functions was defined by Kaplan [19], where the following geometrical interpretation was given: $f \in \mathcal{A}$ is close-to-convex if, and only if, there are no sections of the curve $f\left(\mathbb{T}_{r}\right)$, for every $r \in(0,1)$, in which tangent vector turns backward through an angle not less then $\pi$ (cf. [16, Vol. II, p. 4]). An analogous geometrical interpretation for close-to-star functions was given by Reade, namely, $f \in \mathcal{A}$ is close-to-star if, and only if, there are no sections of the curve $f\left(\mathbb{T}_{r}\right)$, for every $r \in(0,1)$, in which radius vector to the curve $f\left(\mathbb{T}_{r}\right)$ turns backward through an angle not less then $\pi$ ([36, p. 61]). Recall also that Lewandowski $[29,30]$ showed that the class of close-to-convex functions is identical with the class of linearly accessible functions introduced by Biernacki [5].

Let us mention that the class $\mathcal{C S} \mathcal{T}$ and its subclasses were examined by various authors (e.g., MacGregor [31], Sakaguchi [38], Causey and Merkes [8]; for further references, see [16, Vol. II, pp. 97-104]).

Given $g \in \mathcal{S}^{*}$ and $\beta \in \mathbb{R}$, let $\mathcal{C S} \mathcal{T}_{\beta}(g)$ be the subclass of $\mathcal{C S} \mathcal{T}$ of all $f$ satisfying (2). The classes $\mathcal{C} \mathcal{S} \mathcal{T}_{0}\left(g_{i}\right), i=1,2,3$, where

$$
g_{1}(z):=\frac{z}{1-z^{2}}, \quad g_{2}(z):=\frac{z}{(1-z)^{2}}, \quad g_{3}(z):=z, \quad z \in \mathbb{D},
$$

are particularly interested and were separately studied by various authors. In this paper we deal with $\mathcal{C S T}_{0}\left(g_{1}\right)=: \mathcal{S T}$ (i) and $\mathcal{C S} \mathcal{T}_{0}\left(g_{2}\right)=: \mathcal{S T}(1)$ which elements $f$ in view of (2) satisfy the condition

$$
\operatorname{Re}\left\{\left(1-z^{2}\right) \frac{f(z)}{z}\right\}>0, \quad z \in \mathbb{D}
$$

and

$$
\operatorname{Re}\left\{(1-z)^{2} \frac{f(z)}{z}\right\}>0, \quad z \in \mathbb{D},
$$

respectively. Let us add that every function $f$ in $\mathcal{S T}$ (i) or in $\mathcal{S} \mathcal{T}$ (1) which is analytic in the closed disk $\overline{\mathbb{D}}$ is starlike in one direction as defined by Robertson [37] (see also [16, Vol. I, pp. 207-208]), i.e., intersection of $f(\mathbb{D})$ with the real axis is a line segment. Moreover, every $F$ given by (3) with $f \in \mathcal{S} \mathcal{T}$ (i) maps univalently $\mathbb{D}$ onto a domain $F(\mathbb{D})$ convex in the direction of the imaginary axis as defined by Roberston [37] (e.g., [16, p. 199]). Every $F$ given by (3) with $f \in \mathcal{S T}(1)$ maps univalently $\mathbb{D}$ onto a domain $F(\mathbb{D})$ called convex in the positive the direction of the real axis, i.e., $\{w+i t: t \geq 0\} \subset f(\mathbb{D})$ for every $w \in f(\mathbb{D})$ $([6,12,13,15,26,27])$. Let us remark that the condition (4) was generalized in [17] by replacing the expression $1-z^{2}$ by $1-\alpha^{2} z^{2}$ with $\alpha \in[0,1]$.

In this paper we compute the sharp upper and lower bounds for

$$
T_{3,1}(f)=\left|\begin{array}{ccc}
1 & a_{2} & a_{3} \\
\bar{a}_{2} & 1 & a_{2} \\
\bar{a}_{3} & \bar{a}_{2} & 1
\end{array}\right|=2 \operatorname{Re}\left(a_{2}^{2} \bar{a}_{3}\right)-2\left|a_{2}\right|^{2}-\left|a_{3}\right|^{2}+1
$$

for $f$ in $\mathcal{S T}$ (i) and $\mathcal{S T}(1)$. 
Let $\mathcal{P}$ be the class of all $p \in \mathcal{H}$ of the form

$$
p(z)=1+\sum_{n=1}^{\infty} c_{n} z^{n}, \quad z \in \mathbb{D},
$$

which have a positive real part in $\mathbb{D}$.

In the proof of the main result we will use the following lemma will which contains the Carahéodory results (8) and (9), and the well-known formula for $c_{2}$ ([7], [33, p. 166] and further remarks in [11]).

Lemma 1 If $p \in \mathcal{P}$ is of the form (7), then

$$
\left|c_{n}\right| \leq 2, \quad n \in \mathbb{N} .
$$

Moreover

$$
c_{1}=2 \zeta_{1}
$$

and

$$
c_{2}=2 \zeta_{1}^{2}+2\left(1-\left|\zeta_{1}\right|^{2}\right) \zeta_{2}
$$

for some $\zeta_{i} \in \overline{\mathbb{D}}, i \in\{1,2\}$.

For $\zeta_{1} \in \mathbb{T}$, there is a unique function $p \in \mathcal{P}$ with $c_{1}$ as in (9), namely,

$$
p(z)=\frac{1+\zeta_{1} z}{1-\zeta_{1} z}, \quad z \in \mathbb{D} .
$$

For $\zeta_{1} \in \mathbb{D}$ and $\zeta_{2} \in \mathbb{T}$, there is a unique function $p \in \mathcal{P}$ with $c_{1}$ and $c_{2}$ as in (9) and (10), namely,

$$
p(z)=\frac{1+\left(\bar{\zeta}_{1} \zeta_{2}+\zeta_{1}\right) z+\zeta_{2} z^{2}}{1+\left(\bar{\zeta}_{1} \zeta_{2}-\zeta_{1}\right) z-\zeta_{2} z^{2}}, \quad z \in \mathbb{D} .
$$

\section{The class $\mathcal{S} \mathcal{T}$ (i)}

Let $f \in \mathcal{S T}$ (i) be the form (1). By (4) there exists $p \in \mathcal{P}$ of the form (7) such that

$$
\left(1-z^{2}\right) \frac{f(z)}{z}=p(z), \quad z \in \mathbb{D} .
$$

Substituting the series (1) and (7) into (12), and equating coefficients we obtain

$$
a_{2}=c_{1}, \quad a_{3}=c_{2}+1 .
$$

Hence and from (8) it follows that $A_{2}(\mathcal{S T}(\mathrm{i}))=2$ with the Koebe function

$$
k(z):=\frac{z}{(1-z)^{2}}=z+\sum_{n=2}^{\infty} n z^{n}, \quad z \in \mathbb{D},
$$

being extremal. Since the identity function belongs to $\mathcal{S T}$ (i), by Theorem 1 we deduce

Theorem 2 If $f \in \mathcal{S T}(\mathrm{i})$, then

$$
-3 \leq T_{2,1}(f) \leq 1
$$

Both inequalities are sharp. 
We next compute the upper and lower bounds of $T_{3,1}(f)$.

Theorem 3 If $f \in \mathcal{S T}$ (i), then

$$
T_{3,1}(f) \leq 8
$$

The inequality is sharp.

Proof By (13) and (8) we see that $\left|a_{2}\right| \leq 2$ and $\left|a_{3}\right| \leq 3$. Since $\operatorname{Re}\left(a_{2}^{2} \bar{a}_{3}\right) \leq\left|a_{2}^{2} a_{3}\right|$, from (6) we obtain

$$
T_{3,1}(f) \leq F\left(\left|a_{2}\right|,\left|a_{3}\right|\right)
$$

where

$$
F(x, y):=2 x^{2} y-2 x^{2}-y^{2}+1, \quad(x, y) \in[0,2] \times[0,3] .
$$

Observe that the point $(1,1)$ is a unique solution in $(0,2) \times(0,3)$ of the system of equations

$$
\begin{aligned}
& \frac{\partial F}{\partial x}=4 x(y-1)=0, \\
& \frac{\partial F}{\partial y}=2\left(x^{2}-y\right)=0 .
\end{aligned}
$$

However

$$
\frac{\partial^{2} F}{\partial x^{2}}(1,1) \frac{\partial^{2} F}{\partial y^{2}}(1,1)-\left(\frac{\partial F}{\partial x \partial y}(1,1)\right)^{2}=-16<0,
$$

so $(1,1)$ is a saddle point of $F$.

On the boundary of $[0,2] \times[0,3]$ we have

1. $F(0, y)=1-y^{2} \leq 1, \quad y \in[0,3]$;

2. $F(2, y)=-7+8 y-y^{2} \leq 8, \quad y \in[0,3]$;

3. $F(x, 0)=1-2 x^{2} \leq 1, \quad x \in[0,2]$;

4. $F(x, 3)=-8+4 x^{\overline{2}} \leq 8, \quad x \in[0,2]$.

Therefore the inequality $F(x, y) \leq 8$ holds for all $(x, y) \in[0,2] \times[0,3]$, which in view of (16) shows (15).

For the Koebe function (14), $a_{2}=2$ and $a_{3}=3$, which makes equality in (15).

Theorem 4 If $f \in \mathcal{S T}$ (i), then

$$
T_{3,1}(f) \geq-9
$$

The inequality is sharp.

Proof Substituting (9) and (10) into (13) we obtain

$$
a_{2}=2 \zeta_{1}, \quad a_{3}=1+2 \zeta_{1}^{2}+2\left(1-\left|\zeta_{1}\right|^{2}\right) \zeta_{2}
$$

for some $\zeta_{i} \in \overline{\mathbb{D}}, i=1,2$. Therefore from (6) we have

$$
T_{3,1}(f)=2\left(\Psi_{1}+\Psi_{2}\right),
$$

where

$$
\Psi_{1}:=-4\left|\zeta_{1}\right|^{2}+6\left|\zeta_{1}\right|^{4}-2\left(1-\left|\zeta_{1}\right|^{2}\right)^{2}\left|\zeta_{2}\right|^{2}
$$

and

$$
\Psi_{2}:=2 \operatorname{Re}\left(\zeta_{1}^{2}\right)-2\left(1-\left|\zeta_{1}\right|^{2}\right) \operatorname{Re} \zeta_{2}+4\left(1-\left|\zeta_{1}\right|^{2}\right) \operatorname{Re}\left(\zeta_{1}^{2} \bar{\zeta}_{2}\right)
$$


(A) If $\zeta_{1}=0$, then

$$
\Psi_{1}=-2\left|\zeta_{2}\right|^{2}, \quad \Psi_{2}=-2 \operatorname{Re}\left(\zeta_{2}\right)
$$

and therefore

$$
T_{3,1}(f)=-4\left(\left|\zeta_{2}\right|^{2}+\operatorname{Re}\left(\zeta_{2}\right)\right) \geq-8
$$

(B) If $\zeta_{2}=0$, then

$$
\Psi_{1}:=-4\left|\zeta_{1}\right|^{2}+6\left|\zeta_{1}\right|^{4}, \quad \Psi_{2}=2 \operatorname{Re}\left(\zeta_{1}^{2}\right),
$$

and therefore

$$
T_{3,1}(f)=4\left[3\left|\zeta_{1}\right|^{4}-2\left|\zeta_{1}\right|^{2}+\operatorname{Re}\left(\zeta_{1}^{2}\right)\right] \geq-3 .
$$

(C) Suppose that $\zeta_{1} \zeta_{2} \neq 0$. Then $\zeta_{1}=r \mathrm{e}^{\mathrm{i} \theta}$ and $\zeta_{2}=s \mathrm{e}^{\mathrm{i} \psi}$, where $r, s \in(0,1]$ and $\theta, \psi \in[0,2 \pi)$. We have

$$
\begin{aligned}
\Psi_{2} & =2 r^{2} \cos 2 \theta-2 s\left(1-r^{2}\right) \cos \psi+4 r^{2} s\left(1-r^{2}\right) \cos (2 \theta-\psi) \\
& =2 r^{2} \sin (2 \theta+\alpha) \sqrt{1+4 s^{2}\left(1-r^{2}\right)^{2}+4 s\left(1-r^{2}\right) \cos \psi}-2 s\left(1-r^{2}\right) \cos \psi,
\end{aligned}
$$

where $\alpha \in \mathbb{R}$ is a quantity satisfying

$$
\cos \alpha=\frac{\kappa_{1}}{\sqrt{\kappa_{1}^{2}+\kappa_{2}^{2}}}, \quad \sin \alpha=\frac{\kappa_{2}}{\sqrt{\kappa_{1}^{2}+\kappa_{2}^{2}}}
$$

with

$$
\kappa_{1}=2 s\left(1-r^{2}\right) \sin \psi, \quad \kappa_{2}=1+2 s\left(1-r^{2}\right) \cos \psi .
$$

Since $\sin (2 \theta+\alpha) \geq-1$ and $\cos \psi \leq 1$, we have

$$
\begin{aligned}
-\Psi_{2} & \leq 2 r^{2} \sqrt{1+4 s^{2}\left(1-r^{2}\right)^{2}+4 s\left(1-r^{2}\right) \cos \psi}+2 s\left(1-r^{2}\right) \cos \psi \\
& \leq 2 r^{2}+2\left(1-r^{2}\right)\left(1+2 r^{2}\right) s .
\end{aligned}
$$

Therefore by (18) and (22) for $(r, s) \in(0,1]^{2}$ we obtain

$$
\frac{1}{4} T_{3,1}(f)=\frac{1}{2}\left(\Psi_{1}+\Psi_{2}\right) \geq F(r, s),
$$

where

$$
F(x, y):=-\left(1-x^{2}\right)\left[3 x^{2}+\left(1+2 x^{2}\right) y+\left(1-x^{2}\right) y^{2}\right], \quad(x, y) \in[0,1]^{2} .
$$

Since

$$
\frac{\partial F}{\partial y}=-\left(1-x^{2}\right)\left[1+2 x^{2}+2\left(1-x^{2}\right) y\right] \leq 0, \quad(x, y) \in[0,1]^{2},
$$

we conclude that

$$
F(x, y) \geq F(x, 1)=-2-2 x^{2}+4 x^{4} \geq F(1 / 2,1)=-9 / 4, \quad(x, y) \in[0,1]^{2} .
$$

Summarizing, the last inequality together with (23), (19), (20) shows (17).

The function

$$
f(z)=\frac{z\left(1+z^{2}\right)}{\left(1-z^{2}\right)\left(1-\mathrm{i} z-z^{2}\right)}, \quad z \in \mathbb{D},
$$

belongs to $\mathcal{S T}$ (i) having $a_{2}=\mathrm{i}$ and $a_{3}=2$, for which equality in (17) holds. 


\section{The class $\mathcal{S T}(1)$}

Let $f \in \mathcal{S T}$ (1) be the form (1). By (5) there exists $p \in \mathcal{P}$ of the form (7) such that

$$
(1-z)^{2} \frac{f(z)}{z}=p(z), \quad z \in \mathbb{D} .
$$

Substituting (1) and (7) into (24), and equating coefficients we obtain

$$
a_{2}=2+c_{1}, \quad a_{3}=3+2 c_{1}+c_{2} .
$$

Hence and from (8) it follows that $A_{2}(\mathcal{S T}(1))=4$ with the extremal function

$$
f(z)=\frac{z(1+z)}{(1-z)^{3}}=\sum_{n=1}^{\infty} n^{2} z^{n}, \quad z \in \mathbb{D} .
$$

Note that the function

$$
f(z)=\frac{z}{1-z^{2}}, \quad z \in \mathbb{D}
$$

for which $a_{2}=0$, is an element of $\mathcal{S T}(1)$. Thus by Theorem 1 we deduce

Theorem 5 If $f \in \mathcal{S T}(1)$, then

$$
-15 \leq T_{2,1}(f) \leq 1
$$

Both inequalities are sharp.

We next compute the upper and lower bounds of $T_{3,1}(f)$.

Theorem 6 If $f \in \mathcal{S} \mathcal{T}(1)$, then

$$
T_{3,1}(f) \leq 176
$$

The inequality is sharp.

Proof By (25) and (8) we see that $\left|a_{2}\right| \leq 4$ and $\left|a_{3}\right| \leq 9$. As in the proof of Theorem 3, the inequality (16) holds with

$$
F(x, y):=2 x^{2} y-2 x^{2}-y^{2}+1, \quad(x, y) \in[0,4] \times[0,9] .
$$

It is easy to check that $F$ has no relative maximum in $(0,4) \times(0,9)$.

On the boundary of $[0,4] \times[0,9]$ we have

1. $F(0, y)=1-y^{2} \leq 1, \quad y \in[0,9]$;

2. $F(4, y)=-31+32 y-y^{2} \leq F(4,9)=176, \quad y \in[0,9]$;

3. $F(x, 0)=1-2 x^{2} \leq 1, \quad x \in[0,4]$;

4. $F(x, 9)=-80+16 x^{2} \leq F(4,9)=176, \quad x \in[0,4]$.

Therefore the inequality $F(x, y) \leq 176$ holds for all $(x, y) \in[0,4] \times[0,9]$ which in view of (16) shows (27).

For the function (26), $a_{2}=4$ and $a_{3}=9$, which makes equality in (27).

Theorem 7 If $f \in \mathcal{S} \mathcal{T}(1)$, then

$$
T_{3,1}(f) \geq \frac{-442+208 \sqrt{3}+20 \sqrt{28+10 \sqrt{3}}-21 \sqrt{84+30 \sqrt{3}}}{9(-1+3 \sqrt{3})}=-5.0829 \ldots
$$

The inequality is sharp. 
Proof Substituting (9) and (10) into (25) we obtain

$$
a_{2}=2\left(1+\zeta_{1}\right), \quad a_{3}=3+4 \zeta_{1}+2 \zeta_{1}^{2}+2\left(1-\left|\zeta_{1}\right|^{2}\right) \zeta_{2},
$$

for some $\zeta_{i} \in \overline{\mathbb{D}}, i=1,2$. Therefore from (6) we have

$$
T_{3,1}(f)=\Psi_{1}+\Psi_{2},
$$

where

$$
\Psi_{1}:=8+40\left|\zeta_{1}\right|^{2}+12\left|\zeta_{1}\right|^{4}-4\left(1-\left|\zeta_{1}\right|^{2}\right)^{2}\left|\zeta_{2}\right|^{2}
$$

and

$$
\begin{aligned}
\Psi_{2}:= & 40 \operatorname{Re} \zeta_{1}+28 \operatorname{Re}\left(\zeta_{1}^{2}\right)+48\left|\zeta_{1}\right|^{2} \operatorname{Re} \zeta_{1}+16\left(1-\left|\zeta_{1}\right|^{2}\right) \operatorname{Re}\left(\zeta_{1} \bar{\zeta}_{2}\right) \\
& +4\left(1-\left|\zeta_{1}\right|^{2}\right) \operatorname{Re} \zeta_{2}+8\left(1-\left|\zeta_{1}\right|^{2}\right) \operatorname{Re}\left(\zeta_{1}^{2} \bar{\zeta}_{2}\right) .
\end{aligned}
$$

(A) Suppose that $\zeta_{1} \zeta_{2} \neq 0$. Thus $\zeta_{1}=r \mathrm{e}^{\mathrm{i} \theta}$ and $\zeta_{2}=s \mathrm{e}^{\mathrm{i} \psi}$, where $r, s \in(0,1]$ and $\theta, \psi \in$ $[0,2 \pi)$. Then

$$
\Psi_{2}=4\left(\Psi_{3}+\Psi_{4}\right)
$$

where

$$
\begin{aligned}
\Psi_{3} & :=10 r \cos \theta+7 r^{2} \cos 2 \theta+12 r^{3} \cos \theta \\
& =-7 r^{2}+10 r \cos \theta+12 r^{3} \cos \theta+14 r^{2} \cos ^{2} \theta
\end{aligned}
$$

and

$$
\Psi_{4}:=4 r s\left(1-r^{2}\right) \cos (\theta-\psi)+s\left(1-r^{2}\right) \cos \psi+2 r^{2} s\left(1-r^{2}\right) \cos (2 \theta-\psi) .
$$

Furthermore, we have

$$
\Psi_{4}=s\left(1-r^{2}\right) \sqrt{\kappa_{1}^{2}+\kappa_{2}^{2}} \sin (\psi+\alpha),
$$

where $\alpha$ is the quantity satisfying (21) with

$$
\kappa_{1}=4 r \sin \theta+2 r^{2} \sin 2 \theta, \quad \kappa_{2}=1+4 r \cos \theta+2 r^{2} \cos 2 \theta .
$$

Since $\sin (\psi+\alpha) \geq-1$ and $s \leq 1$, we have

$$
\begin{aligned}
\Psi_{4} & \geq-\left(1-r^{2}\right) \sqrt{\kappa_{1}^{2}+\kappa_{2}^{2}} \\
& =-\left(1-r^{2}\right) \sqrt{1+12 r^{2}+4 r^{4}+8 r \cos \theta+16 r^{3} \cos \theta+8 r^{2} \cos ^{2} \theta} .
\end{aligned}
$$

Therefore from (31), (32) and (33) we obtain

$$
\begin{aligned}
\frac{1}{4} \Psi_{2}= & \Psi_{3}+\Psi_{4} \geq 10 r \cos \theta+7 r^{2} \cos 2 \theta+12 r^{3} \cos \theta \\
& -\left(1-r^{2}\right) \sqrt{1+12 r^{2}+4 r^{4}+8 r \cos \theta+16 r^{3} \cos \theta+8 r^{2} \cos ^{2} \theta} .
\end{aligned}
$$

Taking into account that $\left|\zeta_{2}\right|=s \leq 1$, from (30) we have

$$
\Psi_{1} \geq 4\left(1+12 r^{2}+2 r^{4}\right) .
$$

Thus from (29), (36) and (37) it follows that

$$
\frac{1}{4}\left|T_{3,1}(f)\right| \geq G(r, \cos \theta), \quad r \in(0,1], \theta \in[0,2 \pi),
$$

where

$$
G(t, x):=g_{1}(t, x)-\left(1-t^{2}\right) \sqrt{g_{2}(t, x)}
$$


with

$$
g_{1}(t, x):=1+5 t^{2}+2 t^{4}+10 t x+12 t^{3} x+14 t^{2} x^{2}
$$

and

$$
g_{2}(t, x):=1+12 t^{2}+4 t^{4}+8 t x+16 t^{3} x+8 t^{2} x^{2}
$$

for $t \in[0,1]$ and $x \in[-1,1]$.

Let $\Omega:=[0,1] \times[-1,1]$ and

$$
\Theta:=\frac{-442+208 \sqrt{3}+20 \sqrt{28+10 \sqrt{3}}-21 \sqrt{84+30 \sqrt{3}}}{36(-1+3 \sqrt{3})}=-1.27073 \ldots
$$

We now show that

$$
\min \{G(t, x):(t, x) \in \Omega\}=\Theta .
$$

(A1) We first find critical points of $G$ in the interior of $\Omega$, i.e., in $(0,1) \times(-1,1)$. From the equation

$$
0=\frac{\partial G}{\partial x}(t, x)=\frac{\partial g_{1}}{\partial x}(t, x)-\frac{1}{2}\left(1-t^{2}\right)\left(g_{2}(t, x)\right)^{-1 / 2} \frac{\partial g_{2}}{\partial x}(t, x),
$$

it follows that

$$
g_{2}(t, x)^{1 / 2}=\frac{\left(1-t^{2}\right) \frac{\partial g_{2}}{\partial x}(t, x)}{2 \frac{\partial g_{1}}{\partial x}(t, x)}=\frac{2\left(1-t^{2}\right)\left(1+2 t^{2}+2 t x\right)}{5+6 t^{2}+14 t x},
$$

or equivalently,

$$
\Phi=\Phi(t, x):=\left(5+6 t^{2}+14 t x\right)^{2} g_{2}(t, x)-4\left(1-t^{2}\right)^{2}\left(1+2 t^{2}+2 t x\right)^{2}=0 .
$$

Furthermore, note that by (39),

$$
0 \leq \frac{\left(g_{2}(t, x)\right)^{1 / 2}}{2\left(1-t^{2}\right)}=\frac{1+2 t^{2}+2 t x}{5+6 t^{2}+14 t x} .
$$

Differentiating $G$ with respect to $t$ yields

$$
\frac{\partial G}{\partial t}(t, x)=\frac{\partial g_{1}}{\partial t}(t, x)+2 t\left(g_{2}(t, x)\right)^{1 / 2}-\frac{1}{2}\left(1-t^{2}\right)\left(g_{2}(t, x)\right)^{-1 / 2} \frac{\partial g_{2}}{\partial t} .
$$

By (39) and (42) we obtain

$$
\begin{aligned}
\left.\frac{\partial g_{1}}{\partial x} \frac{\partial g_{2}}{\partial x} \frac{\partial G}{\partial t}\right|_{(t, x)} & =\frac{\partial g_{1}}{\partial t} \frac{\partial g_{1}}{\partial x} \frac{\partial g_{2}}{\partial x}+t\left(1-t^{2}\right)\left(\frac{\partial g_{2}}{\partial x}\right)^{2}-\left.\left(\frac{\partial g_{1}}{\partial x}\right)^{2} \frac{\partial g_{2}}{\partial t}\right|_{(t, x)} \\
& =-128 r^{3} H(t, x),
\end{aligned}
$$

where

$$
H(t, x):=\left(3+4 t^{2}+8 t x\right)\left(4+5 t^{2}+2 t^{4}+17 t x+8 t^{3} x+14 t^{2} x^{2}\right) .
$$

Therefore each critical point of $G$ satisfies

$$
3+4 t^{2}+8 t x=0
$$

or

$$
4+5 t^{2}+2 t^{4}+\left(17 t+8 t^{3}\right) x+14 t^{2} x^{2}=0 .
$$


(A1.1.) Assume that (43) holds. Then $x=x(t)=-\left(3+4 t^{2}\right) /(8 t)$, and by (40) we see that

$$
\Phi\left(t,-\frac{3+4 t^{2}}{8 t}\right)=-\frac{1}{32}\left(1+4 t^{2}\right)^{2}\left(39-104 t^{2}+48 t^{4}\right)=0
$$

occurs when $t=\hat{t}_{1}$, where

$$
\hat{t}_{1}=\frac{1}{2} \sqrt{\frac{1}{3}(13-2 \sqrt{13})}=0.694556 \cdots
$$

Thus

$$
x=x\left(\hat{t}_{1}\right)=\hat{x}_{1}=-\frac{1}{78}(13+\sqrt{13}) \sqrt{39-6 \sqrt{13}}=-0.887191 \cdots .
$$

However, it can be seen that

$$
\frac{1+2 \hat{t}_{1}^{2}+2 \hat{t}_{1} \hat{x}_{1}}{5+6 \hat{t}_{1}^{2}+14 \hat{t}_{1} \hat{x}_{1}}<0,
$$

which means that the inequality (41) is not satisfied for $t=\hat{t}_{1}$ and $x=\hat{x}_{1}$. Therefore $G$ has no critical point in the interior of $\Omega$ in the case of (43).

(A1.2.) Suppose now that (44) is satisfied. Then the division algorithm gives $\Phi=0$ when $\tilde{\Phi}=0$, where

$$
\tilde{\Phi}(t, x):=-53+38 t^{2}+312 t^{4}+48 t^{6}-576 t^{8}+t\left(-150+320 t^{2}+496 t^{4}-960 t^{6}\right) x .
$$

On the other hand, (44) as a quadratic equation of $x$ with $\Delta:=65-8 t^{2}-48 t^{4}>$ $0, t \in(0,1)$, has two roots, namely,

$$
x_{i}=x_{i}(t)=\frac{-\left(17+8 t^{2}\right)+(-1)^{i+1} \sqrt{65-8 t^{2}-48 t^{4}}}{28 t}, \quad i=1,2 .
$$

(I) Let $x=x_{1}$. Then $\tilde{\Phi}\left(t, x_{1}(t)\right)=0$ is equivalent to

$$
\begin{gathered}
\left(75-160 t^{2}-248 t^{4}+480 t^{6}\right) \sqrt{65-8 t^{2}-48 t^{4}} \\
=533-1588 t^{2}-1128 t^{4}+6848 t^{6}-4224 t^{8} .
\end{gathered}
$$

Squaring both sides of (46) we obtain

$$
6272 \gamma_{1}(t) \gamma_{2}^{2}(t)=0,
$$

where

$$
\gamma_{1}(t):=-13+12 t^{2}+72 t^{4}, \quad t \in(0,1),
$$

and

$$
\gamma_{2}(t):=1+t^{2}-10 t^{4}+8 t^{6}=\left(1-t^{2}\right)\left(1-2 t^{2}\right)\left(1+4 t^{2}\right), \quad t \in(0,1) .
$$

By finding all zeros of polynomials $\gamma_{1}$ and $\gamma_{2}$ we state that there are two roots $t_{1}$ and $t_{2}$ in $(0,1)$ of the Eq. (47), namely,

$$
t_{1}:=\frac{1}{\sqrt{2}}=0.707 \cdots, \quad t_{2}:=\frac{1}{2} \sqrt{\sqrt{3}-\frac{1}{3}}=0.591 \cdots
$$


For $t=t_{1}$, by (45) we have $\tilde{x}_{1}:=x_{1}\left(t_{1}\right)=-1 / \sqrt{2}$. However, $\tilde{\Phi}\left(t_{1}, \tilde{x}_{1}\right)=$ $7 \neq 0$. For $t=t_{2}$, by (45) we have

$$
\tilde{x}_{2}:=x_{1}\left(t_{2}\right)=\frac{-7+\sqrt{3}}{2 \sqrt{-3+9 \sqrt{3}}}=-0.742378 \cdots
$$

It can be verified that $\tilde{\Phi}\left(t_{2}, \tilde{x}_{2}\right)=0$ and the inequality (41) holds for $t=t_{2}$ and $x=\tilde{x}_{2}$. Therefore $G$ has a critical point at $\left(t_{2}, \tilde{x}_{2}\right)$.

II. If $x=x_{2}$, then a similar method as in the case of $x=x_{1}$ leads us to state that $G\left(t, x_{2}(t)\right)$ is not critical point of $G$ in the interior of $\Omega$ for every $t \in(0,1)$.

Therefore, by A, B1 and B2, the function $G$ has the unique critical point at $\left(t_{2}, \tilde{x}_{2}\right)$. A numerical computation yields

$$
\lambda_{1} \lambda_{3}-\lambda_{2}^{2}=87.8004 \cdots>0 \text { and } \lambda_{1}=8.99071 \cdots>0,
$$

where

$$
\lambda_{1}:=\frac{\partial^{2} G}{\partial t^{2}}\left(t_{2}, \tilde{x}_{2}\right), \quad \lambda_{2}:=\frac{\partial^{2} G}{\partial t \partial x}\left(t_{2}, \tilde{x}_{2}\right), \quad \lambda_{3}=\frac{\partial^{2} G}{\partial x^{2}}\left(t_{2}, \tilde{x}_{2}\right) .
$$

Thus $G$ has a local minimum at $\left(t_{2}, \tilde{x}_{2}\right)$.

(A2.) It remains to consider $G$ on the boundary of $\Omega$.

1. On the side $t=0$,

$$
G(0, x) \equiv 0>\Theta, \quad x \in[-1,1]
$$

2. On the side $t=1$,

$$
G(1, x)=8+22 x+14 x^{2} \geq G(1,-11 / 14)=-9 / 14>\Theta, \quad x \in[-1,1] .
$$

3. On the side $x=1$,

$$
G(t, 1)=2 t\left(3+9 t+8 t^{2}+2 t^{3}\right) \geq G(0,0)=0>\Theta, \quad t \in[0,1] .
$$

4.. On the side $x=-1$,

$$
\varrho(t):=G(t,-1)=1-10 t+19 t^{2}-12 t^{3}+2 t^{4}-\left(1-t^{2}\right)\left|1-4 t+2 t^{2}\right| .
$$

For $t \in[0,(2-\sqrt{2}) / 2]=: I_{1}$ we have $1-4 t+2 t^{2} \geq 0$. Therefore using inequalities

$$
t(1-t) \leq \frac{1}{2}(-1+\sqrt{2}), \quad 3-6 t+2 t^{2} \leq 3, \quad t \in I_{1}
$$

we obtain

$$
\varrho(t)=-2 t(1-t)\left(3-6 t+2 t^{2}\right) \geq 3-3 \sqrt{2}=-1.24264 \cdots>\Theta, \quad t \in I_{1} .
$$

For $t \in[(2-\sqrt{2}) / 2,1]=: I_{2}$ we have $1-4 t+2 t^{2} \leq 0$ and $\varrho(t)=2-14 t+$ $20 t^{2}-8 t^{3}$. Since $\varrho^{\prime}(t)=0$ occurs only when $t=1 / 2 \in I_{2}$ and $\varrho^{\prime \prime}(1 / 2)=$ $16>0$, we obtain

$$
\varrho(t) \geq \varrho(1 / 2)=-1>\Theta, \quad t \in I_{2} .
$$


(B) If $\zeta_{1}=0$, then

$$
\Psi_{1}=8-4\left|\zeta_{2}\right|^{2}, \quad \Psi_{2}=4 \operatorname{Re}\left(\zeta_{2}\right)
$$

and therefore

$$
T_{3,1}(f)=8-4\left|\zeta_{2}\right|^{2}+4 \operatorname{Re}\left(\zeta_{2}\right) \geq 0 .
$$

(C) If $\zeta_{2}=0$ and $\zeta_{1} \neq 0$, then $\Psi_{4}=0$ in view of (33). Thus the inequality (35) is true and therefore further consideration of Part A of the proof remains valid.

Summarizing, from Parts A-C it follows that the inequality (28) holds.

Now, we discuss sharpness. From (29), (36), (37) and (38) we see that $T_{3,1}(f)=4 \Theta$ holds when

$$
r=t_{2}, \quad \cos \theta=\tilde{x}_{2}, \quad s=1, \quad \sin (\psi+\alpha)=-1,
$$

where $t_{2}$ and $\tilde{x}_{2}$ are given by (48) and (49), and $\alpha$ is determined by the condition (21) with $\kappa_{1}$ and $\kappa_{2}$ given in (34). Set $\theta=\arccos \left(\tilde{x}_{2}\right)$, so that it satisfies the second condition in (50). Then $\kappa_{1}=0.889045 \cdots>0$ and $\kappa_{2}=-0.0684473 \cdots<0$. Thus (21) is satisfied if we take

$$
\alpha=-\arccos \left(\frac{\kappa_{1}}{\sqrt{\kappa_{1}^{2}+\kappa_{2}^{2}}}\right)=-0.656114 \cdots
$$

Thus, if we put

$$
\psi=\frac{3 \pi}{2}-\alpha=5.3685 \cdots,
$$

then $\psi$ satisfies the fourth condition in (50). Now, consider a function $\tilde{p}$ which has the form (11) with $\zeta_{1}=t_{2} \mathrm{e}^{\mathrm{i} \theta}$ and $\zeta_{2}=\mathrm{e}^{\mathrm{i} \psi}$. Since $\zeta_{1} \in \mathbb{D}$ and $\zeta_{2} \in \mathbb{T}$, in view of Lemma 1 , we see that $\tilde{p}$ belongs to $\mathcal{P}$. Finally, define

$$
\tilde{f}(z)=\frac{z \tilde{p}(z)}{(1-z)^{2}}, \quad z \in \mathbb{D} .
$$

Since $\tilde{f} \in \mathcal{S T}(1)$ and $T_{3,1}(\tilde{f})=4 \Theta$, the proof of Theorem 7 is completed.

Remark 1 Let us mention that in [14] it was proved that $-3 \leq T_{2,1}(f) \leq 1$ and $-3 \leq$ $T_{3,1}(f) \leq 8$ for $f \in \mathcal{S}^{*}$. Note that the result for $T_{2,1}(f)$ in $\mathcal{S}^{*}$ is identical as for the class $\mathcal{C S T}$ (i) shown in this paper. This is due to the fact that Koebe function $k$ belongs to both classes and is extremal. The difference between these two classes is visible on $T_{3,1}(f)$ which for the class $\mathcal{C S} \mathcal{T}$ (i) is estimated as $-9 \leq T_{3,1}(f) \leq 8$. This is one reason why studying the Hermitian Toeplitz determinants seems sensible. Their lower and upper sharp bounds carry some information about the richness of the class. Classical estimates of coefficients does not necessarily include such a distinction, e.g., both in the class $\mathcal{S}^{*}$ and in $\mathcal{C S T}$ (i) modulus of $n$-th coefficient is bounded by $n$ with $k$ as the extremal function (e.g., [16, Vol. I, p. 116] for the class $\mathcal{S}^{*}$; for the class $\mathcal{C S T}$ (i) it is an immediate consequence of Theorem 16 of [16]).

Acknowledgements We would like to express gratitude to the referees for their constructive comments that helped to improve the clarity of this manuscript. The fifth author was supported by the National Research Foundation of Korea(NRF) grant funded by the Korea government(MSIP; Ministry of Science, ICT \& Future Planning) (No. NRF-2017R1C1B5076778). 
Open Access This article is licensed under a Creative Commons Attribution 4.0 International License, which permits use, sharing, adaptation, distribution and reproduction in any medium or format, as long as you give appropriate credit to the original author(s) and the source, provide a link to the Creative Commons licence, and indicate if changes were made. The images or other third party material in this article are included in the article's Creative Commons licence, unless indicated otherwise in a credit line to the material. If material is not included in the article's Creative Commons licence and your intended use is not permitted by statutory regulation or exceeds the permitted use, you will need to obtain permission directly from the copyright holder. To view a copy of this licence, visit http://creativecommons.org/licenses/by/4.0/.

\section{References}

1. Alexander, J.W.: Functions which map the interior of the unit circle upon simple regions. Ann. Math. 1(7), 12-22 (1915)

2. Ali, Md Firoz, Thomas, D.K., Vasudevarao, A.: Toeplitz determinants whose elements are the coefficients of analytic and univalent functions. Bull. Aust. Math. Soc. 97(2), 253-264 (2018)

3. Babalola, K.O.: On $\mathrm{H}_{3}$ (1) Hankel determinants for some classes of univalent functions. In: Cho, Y.J. (ed.) Inequality Theory and Applications, vol. 6, pp. 1-7. Nova Science Publishers, New York (2010)

4. Bansal, D., Maharana, S., Prajapat, J.K.: Third order Hankel determinant for certain univalent functions. J. Korean Math. Soc. 52(6), 1139-1148 (2015)

5. Biernacki, M.: Sur la représentation conforme des domaines linéairement accessibles. Prace Mat.-Fiz. 44, 293-314 (1936)

6. Bshouty, D., Lyzzaik, A.: Univalent Functions Starlike with Respect to a Boundary Point. Contemp. Math. 382, 83-87 (2005). [Complex Analysis and Dynamical Systems II, A Conference in Honor of Professor Lawrence Zalcman's Sixtieth Birthday, June 9-12, 2003 Nahariya, Israel]

7. Carathéodory, C.: Über den Variabilitatsbereich der Koeffizienten von Potenzreihen, die gegebene werte nicht annehmen. Math. Ann. 64, 95-115 (1907)

8. Causey, W.M., Merkes, E.P.: Radii of starlikeness of certain classes of analytic functions. J. Math. Anal. Appl. 31, 579-586 (1970)

9. Cho, N.E., Kowalczyk, B., Kwon, O.S., Lecko, A., Sim, Y.J.: Some coefficient inequalities related to the Hankel determinant for strongly starlike functions of order alpha. J. Math. Inequal. 11(2), 429-439 (2017)

10. Cho, N.E., Kowalczyk, B., Kwon, O.S., Lecko, A., Sim, Y.J.: The bounds of some determinants for starlike functions of order alpha. Bull. Malays. Math. Sci. Soc. 41(1), 523-535 (2018)

11. Cho, N.E., Kowalczyk, B., Lecko, A.: Sharp bounds of some coefficient functionals over the class of functions convex in the direction of the imaginary axis. Bull. Aust. Math. Soc. 100, 86-96 (2019)

12. Ciozda, K.: O klasie funkcji wypukłych w ujemnym kierunku osi rzeczywistej, jej podklasach i podstawowych własnościach, Ph.D. Thesis, Lublin (1978) (in Polish)

13. Ciozda, K.: Sur la classe des fonctions convexes vers l'axe réel négatif. Bull. Acad. Polon. Sci. 27(3-4), 255-261 (1979)

14. Cudna, K., Kwon, O.S., Lecko, A., Sim, Y.J., Śmiarowska, B.: The second and third-order Hermitian Toeplitz determinants for starlike and convex functions of order $\alpha$. Bol. Soc. Mat. Mex. 2019, 3 (2019). https://doi.org/10.1007/s40590-019-00271-1

15. Elin, M., Shoikhet, D.: Linearization Models for Complex Dynamical Systems. Topics in Univalent Functions, Functional Equations and Semigroup Theory. Birkhäuser, Basel (2010)

16. Goodman, A.W.: Univalent Functions. Mariner, Tampa (1983)

17. Jakubowski, Z.J., Lecko, A.: On certain classes of quasi-typically-real functions. Demonstr. Math. 27(2), 521-536 (1994)

18. Janteng, A., Halim, S.A., Darus, M.: Hankel determinant for starlike and convex functions. Int. J. Math. Anal. 1(13), 619-625 (2007)

19. Kaplan, W.: Close to convex schlicht functions. Michigan Math. J. 1, 169-185 (1952)

20. Kowalczyk, B., Kwon, O.S., Lecko, A., Sim, Y.J.: The bounds of some determinants for functions of bounded turning of order alpha. Bull. Soc. Sci. Lett. Łódź Sér. Rech. Déform. LXVII(1), 107-118 (2017)

21. Kowalczyk, B., Kwon, O.S., Lecko, A., Sim, Y.J., Śmiarowska, B.: The third-order Hermitian Toeplitz determinant for classes of functions convex in one direction. Bull. Malays. Math. Sci. Soc. 2019, 1-16 (2019). https://doi.org/10.1007/s40840-019-00859-w

22. Kowalczyk, B., Lecko, A., Sim, Y.J.: The sharp bound of the Hankel determinant of the third kind for convex functions. Bull. Aust. Math. Soc. 97, 435-445 (2018) 
23. Kowalczyk, B., Lecko, A., Lecko, M., Sim, Y.J.: The sharp bound of the third Hankel determinant for some classes of analytic functions. Bull. Korean Math. Soc. 55(6), 1859-1868 (2018)

24. Kwon, O.S., Lecko, A., Sim, Y.J.: The bound of the Hankel determinant of the third kind for starlike functions. Bull. Malays. Math. Sci. Soc. 42, 767-780 (2019)

25. Lecko, A., Sim, Y.J., Smiarowska, B.: The sharp bound of the Hankel determinant of the third kind for starlike functions of order 1/2. Complex Anal. Oper. Theory 13, 2231-2238 (2019)

26. Lecko, A.: On the class of functions convex in the negative direction of the imaginary axis. J. Aust. Math. Soc. 73, 1-10 (2002)

27. Lecko, A.: Some Methods in the Theory of Univalent Functions. Oficyna Wydawnicza Politechniki Rzeszowskiej, Rzeszów (2005)

28. Lee, S.K., Ravichandran, V., Supramanian, S.: Bound for the second Hankel determinant of certain univalent functions. J. Inequal. Appl. 2013(281), 1-17 (2013)

29. Lewandowski, Z.: Sur l'identiteé de certaines classes de fonctions univalentes, I, II. Ann. Univ. Mariae Curie-Skłodowska Sect. A 12, 131-146 (1958)

30. Lewandowski, Z.: Sur l'identiteé de certaines classes de fonctions univalentes, I, II. Ann. Univ. Mariae Curie-Skłodowska Sect. A 14, 19-46 (1960)

31. MacGregor, T.H.: The radius of univalence of certain analytic functions. Proc. Am. Math. Soc. 14, 514520 (1963)

32. Mishra, A.K., Gochhayat, P.: Second Hankel determinat for a class of analytic functions defined by fractional derivative. Int. J. Math. Math. Sci. 2008, 1-10 (2008). (Article ID 153280)

33. Pommerenke, C.: Univalent Functions. Vandenhoeck \& Ruprecht, Göttingen (1975)

34. Prajapat, J.K., Bansal, D., Singh, A., Mishra, A.K.: Bounds on third Hankel determinant for close-toconvex functions. Acta Univ. Sapientae Math. 7(2), 210-219 (2015)

35. Ravichandran, V., Verma, S.: Generalized Zalcman conjecture for some classes of analytic functions. J. Math. Anal. Appl. 450(1), 592-605 (2017)

36. Reade, M.: On close-to-convex univalent functions. Michigan Math. J. 3, 59-62 (1955)

37. Robertson, M.S.: Analytic functions star-like in one direction. Am. J. Math. 58, 465-472 (1936)

38. Sakaguchi, K.: The radius of convexity for certain class of regular functions. J. Nara Gakugei Univ. Natur. Sci. 12, 5-8 (1964)

39. Sudharsan, T.V., Vijayalaksmi, S.P., Sthephen, B.A.: Third Hankel determinant for a subclass of analytic functions. Malay. J. Mat. 2(4), 438-444 (2014)

Publisher's Note Springer Nature remains neutral with regard to jurisdictional claims in published maps and institutional affiliations. 